\title{
Potencial forrajero de guaje (Leucaena leucocephala) a diferentes densidades de plantas y edades de rebrote
}

\section{Forage potential of guaje (Leucaena leucocephala) at different densities plants and age of growth}

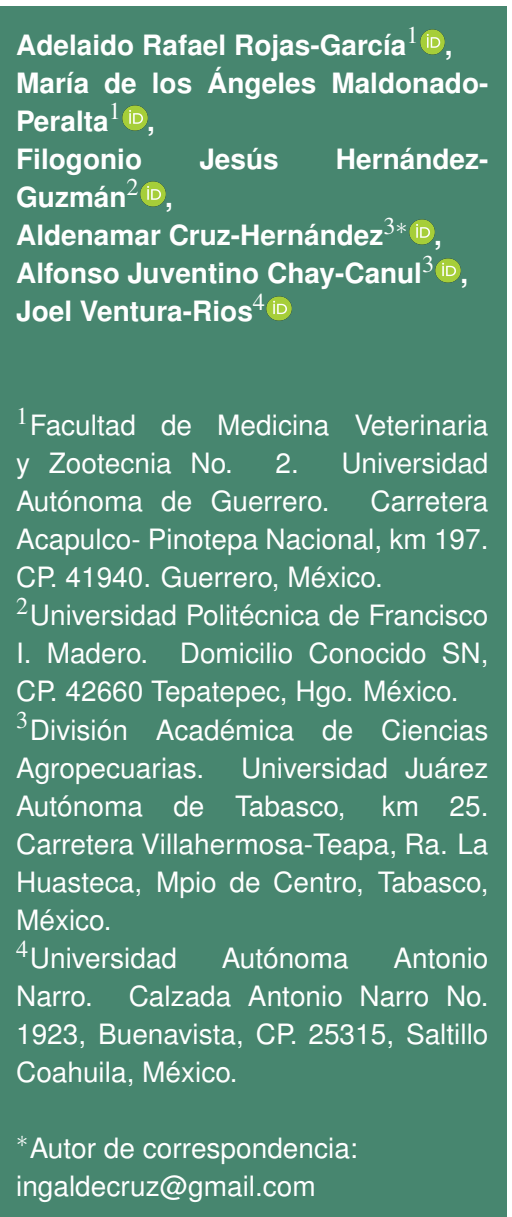

Artículo científico

Recibido: 27 de febrero 2021

Aceptado: 17 de septiembre 2021

Como citar: Rojas-García AR, Maldonado-Peralta MA, HernándezGuzmán FJ, Cruz-Hernández A, Chay-Canul AJ, Ventura-Rios J (2021) Potencial forrajero de guaje (Leucaena leucocephala) a diferentes densidades de plantas y edades de rebrote. Ecosistemas y Recursos Agropecuarios Núm. Esp. II: e2919. DOI: 10.19136/era.a8nll.2919
RESUMEN. Debido a los costos elevados de alimentos concentrados, es necesario promover la utilización de fuentes alimenticias que sean económicas y de elevado valor nutritivo, que ayuden a maximizar la producción animal. El objetivo fue evaluar el comportamiento productivo del guaje (Leucaena leucocephala) a diferentes densidades de plantas y edades de rebrotes en el trópico seco. El estudio se realizó en la época de lluvias (junio a octubre de 2017) en la Facultad de Medicina Veterinaria No. 2 de la Universidad Autónoma de Guerrero en Cuajinicuilapa, Guerrero, México. El guaje (Leucaena leucocephala) se trasplantó a una distancia de $10 \mathrm{~cm}$ entre plantas y entre surcos de 1.5, 2 y $3 \mathrm{~m}$, para obtener densidades de 67, 50 y 33 mil plantas ha ${ }^{-1}$, respectivamente. 30 días antes del inicio de la investigación se realizó una poda de homogenización a $1 \mathrm{~m}$ de altura y posteriormente se realizaron seis estadios de corte cada 15 días (30, 45, 60, 75, 90 y 105 días). El mayor rendimiento de materia seca se obtuvo en la edad de rebrote de 105 días con $7627 \mathrm{~kg}$ MS $\mathrm{ha}^{-1}$ y una densidad de 50000 plantas ha ${ }^{-1}(\mathrm{P} \leq 0.05)$. La densidad de plantas y edad de rebrote óptima en Leucaena fue de 50000 plantas ha $^{-1}$ y 90 dias de rebrote respectivamente, debido a que se presentaron las mejores características estructurales y que coincidió con el 95\% de radiación interceptada.

Palabras clave: Leguminosa, edades de rebrote, densidad de planta, rendimiento.

ABSTRACT. Due to the high costs of concentrated foods, it is necessary to promote the use of food sources that are economical and of high nutritional value, which help to maximize animal production. The objective was to evaluate the productive behavior of the guaje (Leucaena leucocephala) at different plant densities and regrowth ages in the dry tropics. The study was conducted in the rainy season (June to October 2017) at the Faculty of Veterinary Medicine No. 2 of the Autonomous University of Guerrero in Cuajinicuilapa, Guerrero, Mexico. The guaje (Leucaena leucocephala) was transplanted at a distance of $10 \mathrm{~cm}$ between plants and between rows of 1.5, 2 and $3 \mathrm{~m}$, to obtain densities of 67,50 and 33 thousand plants ha ${ }^{-1}$, respectively. 30 days before the beginning of the investigation, a homogenization pruning was carried out at $1 \mathrm{~m}$ height and subsequently six cutting stages were carried out every 15 days $(30,45,60,75,90$ and 105 days). The highest dry matter yield was obtained at the regrowth age of 105 days with 7627 $\mathrm{kg} \mathrm{DM} \mathrm{ha}^{-1}$ and a density of 50000 plants ha $^{-1}(\mathrm{P} \leq 0.05)$. The plant density and optimal regrowth age in Leucaena was 50000 plants ha $^{-1}$ and 90 regrowth days respectively, due to the fact that the best structural characteristics were presented and that considered with $95 \%$ of intercepted radiation.

Key words: Legume, regrowth ages, plant density, yield. 


\section{INTRODUCCIÓN}

Los sistemas de producción agropecuarios en los trópicos tienen la necesidad de incorporar especies que permitan mejorar la productividad y calidad del forraje que se ofrece a los animales (Martínez-Hernández et al. 2019). Generalmente los sistemas que utilizan gramíneas como principal fuente de alimentación presentan limitantes, como la baja calidad del forraje, producción irregular, limitando el correcto funcionamiento ruminal y la producción animal (Izaguirre y Martínez 2008). Debido a los costos elevados de alimentos concentrados, es necesario promover la utilización de fuentes alimenticias que sean económicas y de excelente valor nutritivo, que ayuden a maximizar la producción animal (Rodríguez y Roncallo 2013). La baja producción animal, usualmente está asociada con la concentración de alimentos y el manejo de los pastos tropicales; por lo anterior existe la necesidad de usar especies forrajeras, especialmente leguminosas, las cuales pueden ser introducidas en las áreas de pastoreo para mejorar la calidad y la producción de materia seca (Zamora et al. 2001, Mayren-Mendoza et al. 2018).

En todas las regiones de México, una de las leguminosas con mayor utilización para la alimentación animal es la alfalfa (Rojas et al. 2019), pero esta especie es de clima templado, por lo que para las regiones tropicales se han buscado alternativas, como guaje (Leucaena leucocephala), que contribuye a la alimentación directamente al incrementar la cantidad y calidad del forraje ingerido, e indirectamente al aportar importantes cantidades de nitrógeno al suelo para promover el crecimiento de las gramíneas (Rojas et al. 2017). Las leguminosas, además de incorporar nitrógeno atmosférico al suelo, contribuyen a la producción animal y hacen rentable los sistemas de producción (García-Ferrer et al. 2015). Estas especies arbustivas presentan tolerancias a las sequías, al manejo y una capacidad de rebrotar en tiempos cortos (García et al. 2009); pero la adopción de estas especies no ha sido como se ha esperado, aunque existen evidencias científicas del uso de las leguminosas forrajeras con resultados positivos (Osechas et al. 2008, García et al. 2009).

El guaje es originario de México y Centro América (Martínez-Hernández et al. 2019); es una planta que se comporta como arbusto y en algunos casos como árbol, se utiliza en praderas asociadas o como bancos de proteína (Rubio y Molina 2016). Se han realizado investigaciones en guaje al variar la edad del rebrote obteniendo un acumulativo máximo a los 74 días de rebrote de 6.8 t MS ha $^{-1}$ con una densidad de 60000 plantas ha ${ }^{-1}$, disminuyendo el rendimiento con menores densidades de plantas (Martínez-Hernández et al. 2019), pero se tienen pocas investigaciones sobre densidad de plantas de guaje en México. Por lo anterior, el objetivo del presente estudio fue evaluar el comportamiento productivo de diferentes densidades de guaje (Leucaena leucocephala) y edad de rebrote. La hipótesis fue que la mejor densidad poblacional de plantas es cuando la parcela tiene dos metros entre surcos y $10 \mathrm{~cm}$ entre plantas, con una densidad de 50000 plantas $\mathrm{ha}^{-1}$, ya que proporcionarán mejores características estructurales de la parcela.

\section{MATERIALES Y MÉTODOS}

\section{Localización}

El estudio se realizó durante la época de lluvias de junio a octubre de 2017, en la Facultad de Medicina Veterinaria No. 2 de la Universidad Autónoma de Guerrero, ubicada en Cuajinicuilapa, Guerrero, México. Localizado en las coordenadas $16^{\circ} 28^{\prime} 28^{\prime \prime}$ n y $98^{\circ} 25^{\prime} 11.27$ " o a 46 metros de altitud, con clima Aw denominado trópico seco (García 2004).

\section{Datos climatológicos}

La temperatura media anual en el periodo de estudio fue de $26.5{ }^{\circ} \mathrm{C}$, con una precipitación de 1 $138 \mathrm{~mm}$, durante la época de lluvias (Figura 1). Los datos climáticos se obtuvieron de la estación agrometeorológica de CONAGUA ubicada a $2000 \mathrm{~m}$ de las parcelas experimentales. 


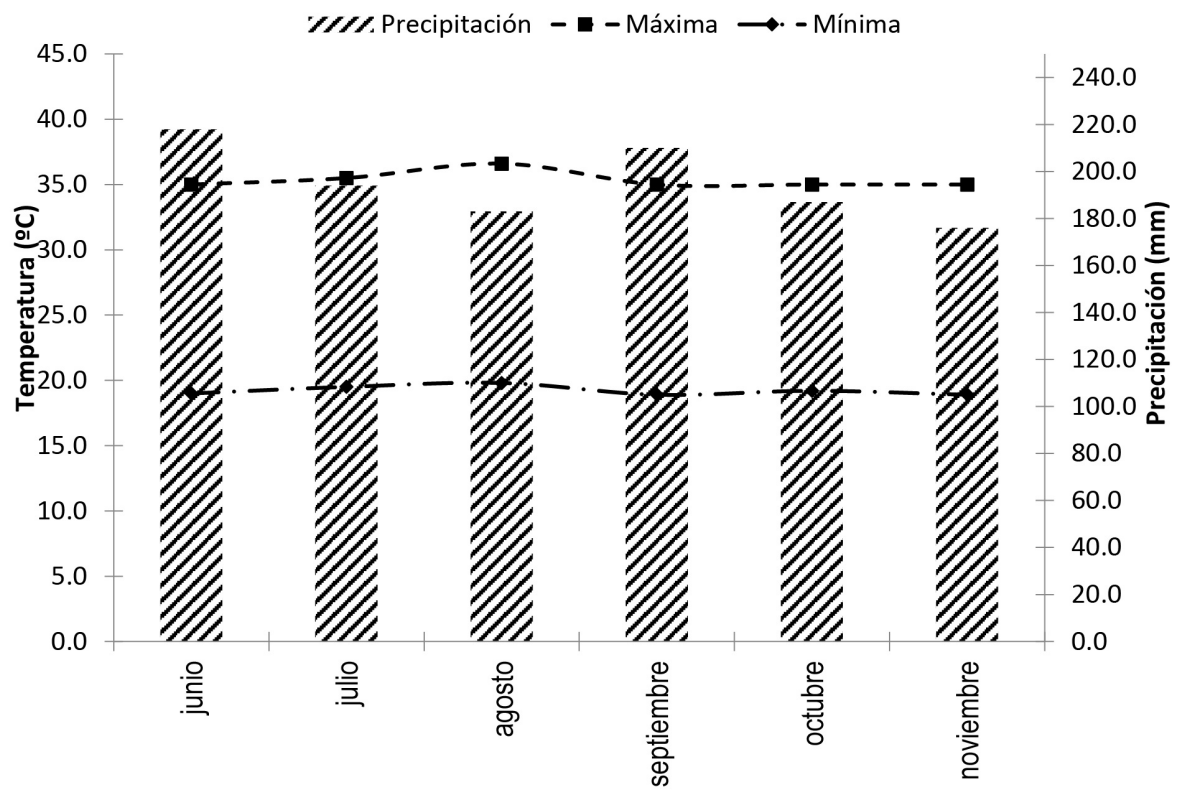

Figura 1. Datos de temperatura máxima, mínima $\left({ }^{\circ} \mathrm{C}\right)$ y precipitación $(\mathrm{mm})$ durante el periodo de estudio de 2017.

\section{Manejo de las parcelas}

La siembra del guaje (Leucaena leucocephala) se realizó en junio de 2016. Se utilizó semilla certificada de Leucaena cv. Cunningham, con $80 \%$ de germinación. Para asegurar una mejor siembra y manejo la semilla se estableció en bolsas de polietileno con capacidad de $1 \mathrm{~kg}$ con sustrato compuesto por $60 \%$ de suelo franco-limoso, $20 \%$ de arena y $20 \%$ de estiércol bovino composteado. Para una pronta germinación y crecimiento se realizaron riegos cada tercer día a capacidad de campo. Cuando los árboles de Leucaena tenían una altura promedio de $1 \mathrm{~m}$ se trasplantaron en parcelas de pasto estrella (Cynodon plectostachyus). La distancia entre plantas de Leucaena fue de $10 \mathrm{~cm}$ y entre surcos de $1.5,2$ y $3 \mathrm{~m}$, para tener las densidades de 67, 50 y 33 mil plantas ha $^{-1}$, respectivamente. Cada unidad experimental fue de $10^{*} 10 \mathrm{~m}$ con tres replicas por cada densidad de siembra. No se fertilizó durante el periodo de estudio. Se realizó una poda de homogenización a una altura de $1 \mathrm{~m}$, a los 30 días antes del inicio de la investigación y posteriormente se realizaron seis cortes cada 15 días (30, 45, 60, 75, 90 y 105 días).

\section{Variables evaluadas}

\section{Rendimiento de materia seca}

Para evaluar el rendimiento de materia seca de Leucaena se realizaron dos muestreos de forma aleatoria en cada parcela útil, de un metro lineal, abarcando 10 plantas por cada muestreo a una altura de $1 \mathrm{~m}$. Se seleccionaron tallos verdes y hojas simulando el ramoneo, posteriormente el forraje cosechado se lavó y se depositó en una estufa de aire forzado a $55^{\circ} \mathrm{C}$ hasta peso constante para estimar la materia seca por hectárea.

\section{Composición morfológica}

De las muestras para el rendimiento de materia seca se tomó el $20 \%$ para evaluar la composición morfológica, mediante la separación de los componentes hojas, tallos verdes y material muerto. Posteriormente cada componente se depositó en bolsas de papel etiquetado y en una estufa de aire forzado a $55{ }^{\circ} \mathrm{C}$ hasta que las muestras obtuvieron peso constante y se registraron. 


\section{Diámetro del tallo y relación hoja: tallo}

El diámetro del tallo se realizó de acuerdo a lo descrito por Rojas et al. (2019) y la relación hoja:tallo se obtuvo a partir de la composición morfológica, al dividir el componente seco de hoja entre tallo. Hoja: tallo $=\mathrm{h} / \mathrm{t}$. Dónde: Hoja: tallo = relación hoja: tallo, $\mathrm{h}=$ peso seco de hoja $\left(\mathrm{kg} \mathrm{MS} \mathrm{ha}^{-1}\right), \mathrm{yt}=$ peso seco de tallo ( $\mathrm{kg} \mathrm{MS} \mathrm{ha}^{-1}$ ).

\section{Altura de la planta}

Un día antes del corte, se tomaron al azar 20 alturas de planta en cada parcela experimental, con una regla graduada en $\mathrm{cm}$, midiendo desde la base del suelo hasta el componente morfológico con mayor altura.

\section{Radiación interceptada}

Un día previo a cada corte, se tomaron al azar cinco lecturas de radiación por repetición, con el método del metro de madera descrito por Rojas et al. (2016) en cada unidad experimental. Las lecturas se realizaron aproximadamente a las 13:00 h (hora para medir la cobertura en el dosel, porque el ángulo solar es alto y la intercepción de la luz cambia al mínimo). Para lo cual se colocó la regla en la superficie del suelo (debajo del dosel), con orientación sur-norte, e inmediatamente después, se contaron los centímetros sombreados, los cuales representan el porcentaje de radiación interceptada por el dosel vegetal.

\section{Análisis estadístico}

Los datos se analizaron mediante un diseño de bloques completamente al azar con arreglo factorial (densidad y edad de rebrote) y tres repeticiones, se utilizó PROC GLM de SAS (2009). La comparación múltiple de medias de los tratamientos se realizó mediante la prueba de Tukey $(\mathrm{P} \leq 0.05)$.

\section{RESULTADOS}

El rendimiento del guaje (Leucaena leucocephala) en tres densidades de plantas y seis edades de rebrote se observa en la Tabla 1 . El crecimiento incrementó con la edad de rebrote hasta alcanzar un punto máximo en todas las densidades. El mayor rendimiento de materia seca se obtuvo a los 105 días con una densidad de 50000 plantas ha $^{-1}(P<0.05)$; mientras que, en las parcelas con densidad de 67000 plantas ha ${ }^{-1}$ que se cosecharon cada 15 días ocurrió lo contrario.

En la Figura 2 se presenta la composición morfológica del guaje en tres densidades de plantas y seis edades de rebrote. Independientemente de la densidad de planta en las primeras edades de rebrote de 30, 45 y 60 días es mayor el porcentaje del componente hoja, con un promedio de $72 \%$; sin embargo, en las ultimas edades aumenta la cantidad de tallo y disminuye la hoja con 40 y $60 \%$, respectivamente $(\mathrm{P}<0.05)$. Con la densidad de 50000 plantas ha $^{-1}$ (Figura 2) se presentó el 69\% de hojas, mayor que las otras densidades, por ende, menor contenido de tallos con $31 \%(P<0,05)$.

Los resultados de diámetro de tallo $(\mathrm{cm})$ y relación hoja:tallo del guaje al variar las edades de rebrote y densidades de plantas $\mathrm{ha}^{-1}$ se presentan en la Tabla 2. El diámetro de tallo fue diferente y se incrementó conforme aumentaron las edades de rebrote, independientemente de la densidad de plantas se obtuvo un diámetro de $0.12 \mathrm{~cm}$ a los 30 días y 1.18 $\mathrm{cm}$ a los 105 días después del corte $(P<0.05)$. La densidad de planta que alcanzó mayor diámetro de tallo fue de 33000 plantas ha ${ }^{-1}$ con 0.14 a $1.24 \mathrm{~cm}$ para el intervalo de 30 a 105 días, respectivamente $(P<0.05)$.

En lo que respecta a la relación hoja:tallo del guaje ocurrió lo contrario a el diámetro de tallo en las primeras edades de rebrote, donde se obtuvo la mayor relación hoja:tallo y disminuyó conforme transcurrió el tiempo de evaluación (Tabla 2). La densidad que presentó la mayor relación hoja:tallo fue la de 50000 plantas ha ${ }^{-1}$, con 3.5 a 30 días del corte y la menor en las densidades de 67000 y 33000 con 1.1 de relación hoja:tallo $(P<0.05)$.

La altura del guaje en diferentes densidades de plantas al variar las edades de rebrote se encuentra en la Tabla 3. Independientemente de la densidad de plantas la altura se incrementó conforme transcurrió el periodo de evaluación $(P<0.05)$. La menor y mayor altura promedio se registró en las densidades 
Tabla 1. Rendimiento de materia seca $\left(\mathrm{kg} \mathrm{MS} \mathrm{ha}^{-1}\right.$ ) del guaje (Leucaena leucocephala) en tres densidades de plantas y seis edades de rebrote.

\begin{tabular}{lrrrrrr}
\hline Densidades (plantas ha ${ }^{-1}$ ) & \multicolumn{7}{c}{ Edades de rebrote (días) } \\
\hline (miles) & 30 & 45 & 60 & 75 & 90 & 105 \\
\hline 67 & $400^{f C}$ & $1400^{e B}$ & $2109^{d B}$ & $2981^{c B}$ & $4821^{b B}$ & $6521^{a B}$ \\
50 & $450^{f B}$ & $1389^{e B}$ & $3029^{d A}$ & $3790^{c A}$ & $5467^{b A}$ & $7627^{a A}$ \\
33 & $574^{f A}$ & $1490^{e A}$ & $1845^{d C}$ & $2890^{c B}$ & $4980^{b B}$ & $6234^{a B}$
\end{tabular}

$\mathrm{abc}=$ Medias con la misma literal minúscula en una misma hilera no son significativas $(P>0.05)$; $A B C=$ Medias con la misma literal mayúscula en una misma columna son significativas $(P>0.05)$.

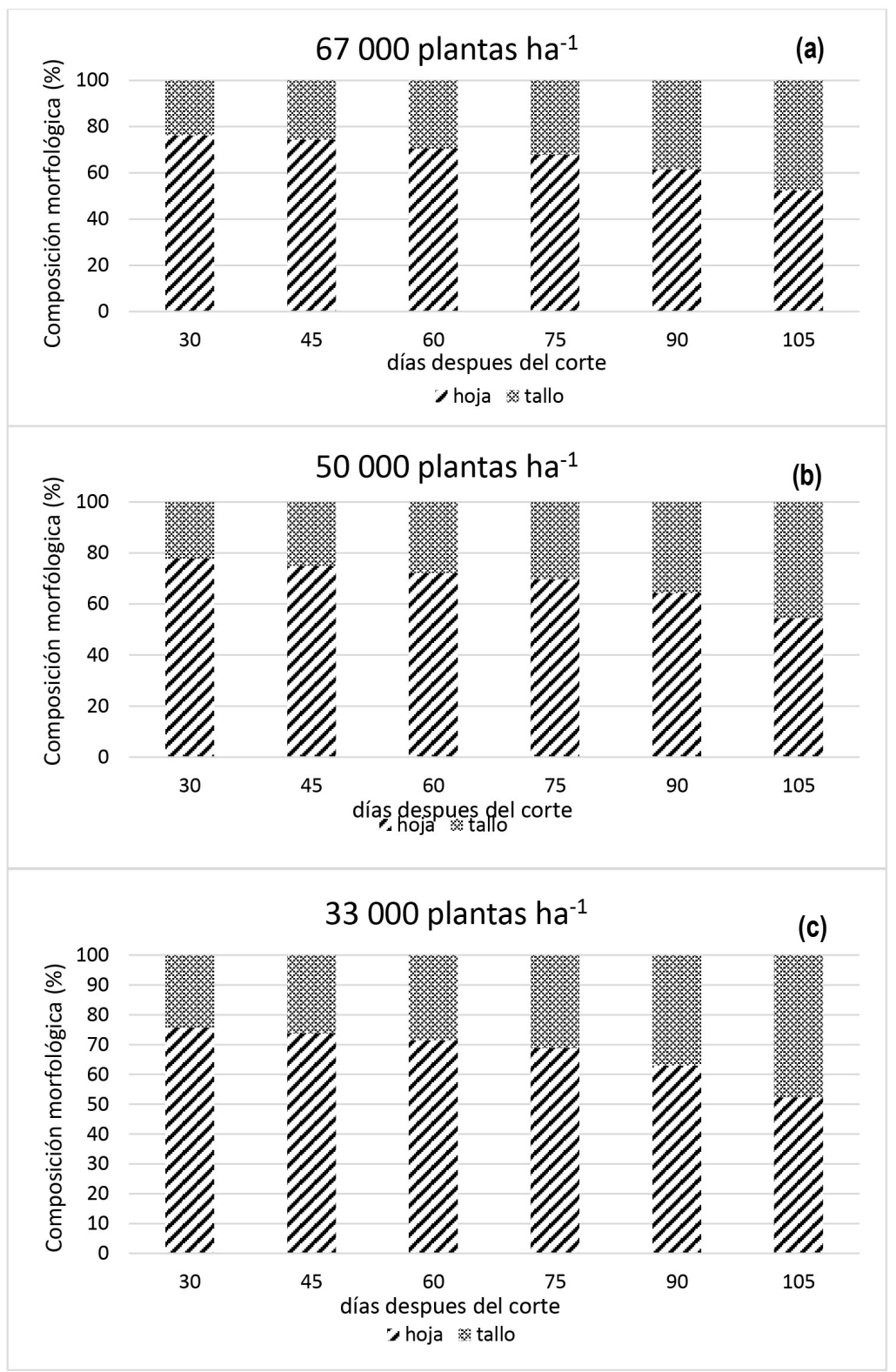

Figura 2. Composición morfológica de la densidad de 67000 (a), 50000 (b) y 33000 (c) plantas ha ${ }^{-1}$ del guaje (Leucaena leucocephala) y seis edades de rebrote. 
Tabla 2. Diámetro de tallo y relación hoja:tallo de guaje (Leucaena leucocephala) en tres densidades al variar las edades de rebrote.

\begin{tabular}{|c|c|c|c|c|c|c|}
\hline \multirow{2}{*}{$\begin{array}{l}\text { Densidades } \\
\text { (plantas ha }^{-1} \text { ) } \\
\text { (miles) }^{\text {mant }}\end{array}$} & \multicolumn{6}{|c|}{$\begin{array}{l}\text { Edades de rebrotes (días) } \\
\text { diámetro de tallo }(\mathrm{cm})\end{array}$} \\
\hline & 30 & 45 & 60 & 75 & 90 & 105 \\
\hline 67 & $0.11^{f B}$ & $0.23^{e B}$ & $0.43^{d B}$ & $0.65^{c B}$ & $0.87^{b B}$ & $1.11^{a C}$ \\
\hline 50 & $0.12^{f B}$ & $0.33^{e A}$ & $0.45^{d B}$ & $0.68^{c B}$ & $0.89^{b B}$ & $1.20^{a B}$ \\
\hline \multirow[t]{2}{*}{33} & $0.14^{f A}$ & $0.34^{e A}$ & $0.54^{d A}$ & $0.73^{c A}$ & $0.92^{b A}$ & $1.24^{a A}$ \\
\hline & \multicolumn{6}{|c|}{ relación hoja:tallo } \\
\hline 67 & $3.2^{a B}$ & $2.9^{b B}$ & $2.4^{c C}$ & $2.1^{d C}$ & $1.6^{e C}$ & $1.1^{F b}$ \\
\hline 50 & $3.5^{a A}$ & $3.1^{b A}$ & $2.6^{c A}$ & $2.3^{c d A}$ & $1.8^{d A}$ & $1.2^{E a}$ \\
\hline 33 & $3.1^{a B}$ & $2.8^{b C}$ & $2.5^{c B}$ & $2.2^{d B}$ & $1.7^{e B}$ & $1.1^{F b}$ \\
\hline
\end{tabular}

Tabla 3. Altura de la planta $(\mathrm{cm})$ de guaje (Leucaena leucocephala) en tres densidades de plantas al variar las edades de rebrote.

\begin{tabular}{lrrrrrr}
\hline Densidades (plantas ha ${ }^{-1}$ ) & \multicolumn{7}{c}{ Edades de rebrote (días) } \\
\hline (miles) & 30 & 45 & 60 & 75 & 90 & 105 \\
\hline 67 & $123^{f B}$ & $154^{e A}$ & $176^{d A}$ & $198^{c A}$ & $210^{b A}$ & $232^{a A}$ \\
50 & $132^{f A}$ & $146^{e B}$ & $165^{d B}$ & $187^{c B}$ & $201^{b B}$ & $228^{a B}$ \\
33 & $123^{f B}$ & $145^{e B}$ & $161^{d B}$ & $189^{c A}$ & $213^{b A}$ & $233^{a A}$ \\
\hline abC = Medias con la misma literal minúscula en una misma hilera son significativas $(\mathrm{P}>$ \\
0.05); ABC = Medias con la misma literal minúscula en una misma columna son signi- \\
ficativas (P > 0.05).
\end{tabular}

de siembra de 67000 y 33000 , con altura de 123 y $233 \mathrm{~cm}$ para las edades de rebrote de 30 y 105 días, respectivamente $(P<0.05)$.

La radiación interceptada en el guaje al variar las edades de rebrote y densidad de plantas se observa en la Tabla 4. Conforme aumentó la edad de rebrote se incrementó la radiación interceptada $(\mathrm{P}<$ 0.05). En la densidad de planta de 67000 plantas $\mathrm{ha}^{-1}$ y edad de rebrote de 90 días fue donde se alcanzó primero el $95 \%$ de la radiación interceptada.

\section{DISCUSIÓN}

El mayor rendimiento de forraje observado en la densidad de 50000 plantas ha $^{-1}$ se puede atribuir a que es la densidad óptima, ya que al aumentar o disminuir la densidad de planta ocurre competencia intra e inter especifica por nutrientes, luz y agua (Lüscher et al. 2014). En este sentido, López-Vigoa et al. (2017) mencionan que los rendimientos de forrajes pueden mantenerse entre 6 y $8 \mathrm{t} \mathrm{MS} \mathrm{ha}^{-1}$ corte $^{-1}$ cuando la altura de corte se mantiene entre
50 y $70 \mathrm{~cm}$ de altura, valores que son similares a los que se obtuvo con 105 días de rebrote. También se tienen reportes que indican la misma tendencia en la cantidad de hoja y tallo en el rebrote de Leucaena similar a esta investigación (Ramírez et al. 2012).

Por otra parte, el resultado con el mayor diámetro de tallos se obtuvo con la menor densidad de siembra, lo cual se puede atribuir a la menor competencia inter específica, por lo que se tuvo mayores recursos de nutrientes, luz y agua por planta; mientras que, la mayor densidad de plantas tuvo dichos recursos en menor cantidad, otro aspecto importante es la edad de la planta (Lestienne et al. 2006, NávarCháidez et al. 2013). La relación hoja:tallo fue variable dependiendo la densidad de siembra y se encuentra relacionado con el mayor rendimiento de materia seca. Existen estudios que reportan que a mayor altura de corte disminuye el forraje de Leucaena, por lo que recomiendan una altura de $80 \mathrm{~cm}$ aproximadamente (Rivas et al. 2009, Román-Miranda et al. 2016).

En lo que respecta a la radiación interceptada Silva et al. (2007) y Carneiro et al. (2008) reportan 
Tabla 4. Radiación interceptada (\%) de guaje (Leucaena leucocephala) en tres densidades de plantas al variar las edades de rebrote.

\begin{tabular}{lcccccc}
\hline Densidades (plantas ha ${ }^{-1}$ ) & \multicolumn{5}{c}{ Edades de rebrote (días) } \\
\hline (miles) & 30 & 45 & 60 & 75 & 90 & 105 \\
\hline 67 & $42^{f A}$ & $54^{e A}$ & $67^{d A}$ & $88^{c A}$ & $95^{b A}$ & $98^{a A}$ \\
50 & $43^{f A}$ & $46^{e B}$ & $65^{d B}$ & $87^{c A}$ & $94^{b A}$ & $95^{a A B}$ \\
33 & $39^{f B}$ & $45^{e B}$ & $61^{d C}$ & $78^{c B}$ & $88^{b B}$ & $90^{a B}$ \\
\hline abc = Medias con la misma literal minúscula en una misma hilera son significativas \\
$(\mathrm{P}>0.05) ; A B C=$ Medias con la misma literal mayúscula en una misma columna \\
son significativas (P > 0.05).
\end{tabular}

que en especies forrajeras tropicales la cosecha debe hacerse cuando se alcance el $95 \%$ de la radiación interceptada, siempre y cuando la densidad de plantas (Mattera et al. 2013), y tallos sean competitivos (Rojas et al. 2017). Por lo que la capacidad de las especies para interceptar la radiación solar depende del número de plantas por hectárea y de las condiciones ambientales, factores que benefician o afectan la radiación, así como el manejo en la parcela (Horrocks y Vallentine 1999, Carneiro et al. 2008).

\section{CONCLUSIONES}

Conforme aumenta la edad de rebrote incrementa el rendimiento, diámetro de tallo, radiación interceptada y altura de la planta de Leucaena. Pero disminuye el componente hoja y la relación hoja:tallo, independientemente de la densidad de plantas. La densidad de plantas y edad de rebrote óptima en guaje fue de 50000 plantas ha $^{-1}$ y 90 días de rebrote con el $95 \%$ de la radiación interceptada.

\section{LITERATURA CITADA}

Carneiro CS, Do Nascimento JD, Batista EVP (2008) Pastagens: Conceitos básicos, produÇão e manejo. Viçosa-MG. Departamento de Zootecnia. Universidade Federal de Viçosa. San Paulo, Brasil. 115p.

García E (2004) Modificaciones al Sistema de Clasificación Climática de Koppen. 4 ed. Universidad Nacional Autónoma de México. México. 217p.

García D, Medina M, Moratinos O, Cova L, Torres A, Santos O, Perdomo D (2009) Caracterización químiconutricional de forrajes leguminosos y de otras familias botánicas empleando análisis descriptivo y multivariado. Avances en Investigación Agropecuaria 13: 25-39.

García-Ferrer AED, Bolaños-Aguilar J, Ramos-Juárez M, Osorio A, Lagunes-Espinoza LC (2015) Rendimiento y valor nutritivo de leguminosas forrajeras en dos épocas del año y cuatro edades de rebrote. Revista Mexicana de Ciencias Pecuarias 6: 453-468.

Horrocks RD, Vallentine JF (1999) Harvested forages. Academic Press. Oval Road, London. United Status of America. 426p.

Izaguirre FF, Martínez TJJ (2008) El uso de árboles multipropósito como alternativa para la producción animal sostenible. Tecnología en Marcha 21: 28-40.

Lestienne F, Thornton B, Gastal F (2006) Impact of defoliation intensity and frequency on N uptake and movilization in Lolium perenne. Journal of Experimental Botany 57: 997-1006.

López-Vigoa O, Sánchez-Santana T, Iglesias-Gómez JM, Lamela-López L, Soca-Pérez M, Arece-García J, MileraRodríguez MC (2017) Los sistemas silvopastoriles como alternativa para la producción animal sostenible en el contexto actual de la ganadería tropical. Pastos y Forrajes 40: 83-95. 
Lüscher A, Mueller-Harvey I, Soussana JF, Reess RM, Peyraud L (2014) Potential of legume-besed grasslandlivestock systems in Europe: a review. Grass and Forage Science 69: 206-228.

Martínez-Hernández PA, Cortez-Díaz E, Purroy-Vázquez R, Palma-García JM, Del Pozo-Rodríguez PP, ViteCristóbal C (2019) Leucaena leucocephala (Lam.) de wit especie clave para una producción bovina sostenible en el trópico. Tropical and Subtropical Agroecosystems 22: 331-357.

Mattera J, Romero LA, Cuatrin AL, Cornaglia PS, Grimoldi AA (2013) Yield components, light interception and radiation use efficiency of lucerne (Medicago sativa L.) in response to row spacing. European Journal of Agronomy 45: 87-95.

Mayren-Mendoza FJ, Rojas-García AR, Maldonado-Peralta MA, Ramírez-Reynoso O, Herrera-Pérez J, TorresSalado N, Sánchez Santillán P, Bottini-Luzardo MB, Hernández-Garay A (2018) Comportamiento productivo de ovinos pelibuey en pastoreo suplementados con follaje de Guazuma ulmifolia Lam. Agroproductividad 11: 29-33.

Návar-Cháidez J, Rodríguez-Flores FD, Domínguez-Calleros PA (2013) Ecuaciones alométricas para árboles tropicales: aplicación al inventario forestal de Sinaloa, México. Agronomía Mesoamericana 24: 347-356.

Osechas D, Ligia B, Rodríguez I (2008) Uso de Leucaena leucocephala como recurso forrajero en fincas doble propósito del estado Trujillo, Venezuela. Agricultura Andina 14: 49-58.

Ramírez M, Suárez H, Regino M, Caraballo B, García DE (2012) Respuesta a tratamientos pregerminativos y caracterización morfológica de plántulas de Leucaena leucocephala, Pithecellobium dulce y Ziziphus mauritiana. Pastos y Forrajes 35: 29-42.

Rivas PF, Castillo HJ, Ortega RL (2009) Selectividad de herbicidas y control de malezas para establecer una asociación Brachiaria brizantha-Leucaena leucocephala. Técnica Pecuaria en México 47: 339-355.

Rodríguez FG, Roncallo FB (2013) Producción de forraje y respuesta de cabras en crecimiento en arreglos silvopastoriles basados en Guazuma ulmifolia, Leucaena leucocephala y Crescentia cujete. Corpoica Ciencia y Tecnologia Agropecucuaria 14: 77-89.

Rojas GAR, Hernández GA, Quero CAR, Guerrero RJD, Ayala W, Zaragoza RJL (2016) Persistencia de Dactylis glomerata L. solo y asociado con Lolium perenne L. y Trifolium repens L. Revista Mexicana de Ciencias Agrícolas 7: 885-895.

Rojas GAR, Hernández GA, Rivas JMA, Mendoza PSI, Maldonado PMA, Joaquín CS (2017) Dinámica poblacional de tallos de pasto ovillo (Dactylis glomerata L.) y ballico perenne (Lolium perenne L.) asociados con trébol blanco (Trifolium repens L.). Revista de la Facultad de Ciencias Agrarias 49: 35-49.

Rojas GAR, Mendoza PSI, Mendoza PMA, Álvarez VP, Torres SN, Cruz HA (2019) Rendimiento de forraje y valor nutritivo de alfalfa a diferentes intervalos de corte. Revista Mexicana de Ciencias Agrícolas 10: 849-858.

Román-Miranda ML, Palma-García JM, Zorrilla-Rios JM, Mora-Santacruz A (2016) Producción de materia seca de Leucaena leucocephala y vegetación herbácea en un banco de proteína pastoreada por ovinos. Revista de Sistemas Experimentales 3: 42-50.

Rubio LA, Molina E (2016) Las leguminosas en la alimentación animal. Arbor 192: 1-14.

SAS (2009) SAS/STAT ${ }^{\circledR}$ 9.2. User's guide release. SAS Institute. Cary, NC, USA. 1848p.

Silva MLO, Faria MA, Morais AR, Andrade GP, Lima EMC (2007) Crescimento e Produtividade do Girassol Cultivado na Entressa fracom Diferentes Lâminas de Água. Revista Brasileira de Engenharia Agrícola e Ambiental 11: 482-488. 
Zamora S, García J, Bonilla G, Aguilar H, AHarvey C, Ibrahim M (2001) Como utilizar los frutos de Guanacaste (Enterolobium cyclocarpum), Guácimo (Guazuma ulmifolia), Genízaro (Pithecellobium saman), y Jícaro (Crescentia alata) en alimentación animal. Agroforestería en las Américas 8: 45-49. 